\title{
Practical indications for the prevention and management of SARS-CoV-2 in ambulatory dialysis patients: lessons from the first phase of the epidemics in Lombardy
}

\author{
Giuseppe Rombolà ${ }^{1}$ Marco Heidempergher ${ }^{2} \cdot$ Luciano Pedrini $^{3} \cdot$ Marco Farina $^{4} \cdot$ Filippo Aucella $^{5}$. \\ Piergiorgio Messa ${ }^{6} \cdot$ Giuliano Brunori $^{7}$
}

Published online: 23 March 2020

(c) Italian Society of Nephrology 2020

\begin{abstract}
Confronting the SARS-CoV-2 outbreak has allowed us to appreciate how efficiently highly-resourced settings can respond to crises. However even such settings are not prepared to deal with the situation, and lessons are only slowly being learnt. There is still an urgent need to accelerate protocols that lead to the implementation of rapid point-of-care diagnostic testing and effective antiviral therapies. In some high-risk populations, such as dialysis patients, where several individuals are treated at the same time in a limited space and overcrowded areas, our objective must be to ensure protection to patients, the healthcare team and the dialysis ward. The difficult Italian experience may help other countries to face the challenges. The experience of the Lombardy underlines the need for gathering and sharing our data to increase our knowledge and support common, initially experience-based, and as soon as possible evidence-based position to face this overwhelming crisis.
\end{abstract}

\section{Background}

The recent infection caused by SARS-CoV-2, a new strand of the Coronavirus, is challenging healthcare systems worldwide. Italy is one of the countries in which the infection has spread most rapidly and where the number of deaths is steadily increasing [1].

Giuseppe Rombolà

giuseppe.rombola@asst-settelaghi.it

1 Sezione Lombarda SIN, U.O. Nefrologia, Dialisi e Trapianto, ASST Sette Laghi, Varese, Italy

2 Sezione Lombarda SIN, U.O. Nefrologia e Dialisi, ASST FBF Sacco, Milan, Italy

3 U.O. Nefrologia e Dialisi, Ospedale Bolognini ASST Bergamo Est, Bergamo, Italy

4 U.O. Nefrologia e Dialisi, ASST Lodi, Lodi, Italy

5 Società Italiana di Nefrologia, U.O. Nefrologia e Dialisi, IRCCS "Casa Sollievo della Sofferenza" San Giovanni Rotondo, Foggia, Italy

6 Società Italiana di Nefrologia-SIN, U.O. Nefrologia, Dialisi e Trapianto, Ospedale Ca Granda Milano, Milan, Italy

7 Società Italiana di Nefrologia-SIN, U.O. Nefrologia e Dialisi, Ospedale Santa Chiara, Trento, Italy
The initial Italian policy has been one of extensive testing of suspected cases including their contacts, and while this may have increased the efficacy of preventive measures, it has contributed to spreading concern. The lack of precise knowledge about the natural history of the disease, the awareness that even non-symptomatic or oligo-symptomatic cases (which probably account for about half of the individuals testing positive for the virus) may spread the infection, and the fact that re-infection is possible, further complicate the situation [2-4].

In common with other viral diseases, mortality is higher in elderly patients with high comorbidity, but no age is spared, and the impressive figures of transmission in different communities underline the need for reorganization of efforts to limit contagion, particularly in crowded settings [5].

Proximity and comorbidity require special attention [6-8]. In this context some populations, such as dialysis patients, combine fragility with the need for care in settings in which several individuals are treated at the same time.

Dialysis patients have a less efficient immune system and are more prone to develop severe infectious diseases than the general population [9-10]. In the case of the SARS-CoV-2, the few data available in the literature referring specifically to dialysis patients suggest that the inflammatory reaction may be less violent and, as a 
consequence, patients on dialysis may have mild clinical signs, at least in the early stages of the disease, with a higher risk of diffusing the infection in the dialysis ward [11].

\section{The Lombardy experience}

In Lombardy, where the first severe infectious outbreak in Italy occurred at the time of this report, two dialysis centers were deeply involved. In the first, a satellite hemodialysis center where 60 patients were being treated, 18 were infected and immediately isolated. About one week elapsed between the first patient and the eighteenth. During this time, the nephrology unit was transformed into an isolation unit and the 18 patients were treated in a small, dedicated dialysis ward set up to deal with the emergency, separated from the main dialysis ward. Of the 18 patients with clinical infection one is in critical condition but so far does not need respiratory assistance. So far none of the healthcare staff has been infected.

The rigidly implemented isolation measures were effective and no other patient (out of a total of about 200 patients on hemodialysis) has developed a clinical picture thereafter. In the second center, $50 \mathrm{~km}$ away, 4 of its 170 patients were found to be infected. They were promptly isolated and no other case has been diagnosed. None of the staff was infected. Both centers are being closely monitored. In both centers the tests were performed only in symptomatic cases; in both units, since the first positive case, all patients have been required to wear surgical masks from the time they arrive in the hemodialysis waiting room and while waiting for swab tests results the healthcare team applies the rules that would be followed if the case were to be confirmed (see below).

\section{Basic, pragmatic suggestions}

To limit the spread of the infection in dialysis centers, it is advisable to act on two fronts: healthcare team and patients (Table 1).

Healthcare team: the goal is to involve and inform the healthcare team so that they do not underestimate the risk of infection, considering that dialysis patients can have subtle manifestations [11]. In this case prevention has multiple objectives: avoiding the spread of infection from patient to patient; preventing nurses and physicians from contracting the infection; avoiding the collapse of healthcare services that would ensue if a high number of healthcare personnel had to be quarantined.

Because healthcare-associated transmission and infection of healthcare workers are a major problem, the Center for Disease Control in the United States recommend that healthcare workers use personal protective equipment (PPE) and implement standard, contact, and airborne precautions including eye protection. The indications are however not fully clear, and may differ across countries [11].

Dialysis patients: The goal is timely identification of affected patients so that they can be isolated, keeping in mind that clinical manifestations can be subtle (general malaise, fatigue, low fever and cold or flu-like manifestations), at least in the early stages of the disease.

The general measures and specific interventions that make it possible to accomplish these goals are set forth below.

\section{General measures}

The first step is to insist that staff observe individual protective measures, which involve rules that should be routine practice in all dialysis centers.

Table 1 The three main goals for limiting the spread of Coronavirus in the dialysis ward

Action 1 Protect patients

Action 2 Protect the team

Action 3 Protect the dialysis ward The dialysis ward is a setting at high risk for infectious outbreaks; the dialysis procedure is time consuming, and the extracorporeal circuit is a risk element; the management of the arteriovenous fistula is likewise critical. The decision to keep positive patients out of the dialysis ward as much as possible, especially at a time when patients are stressed, nurses are working under pressure, and members of the healthcare team may be ill or in quarantine, protects the dialysis ward and makes it possible to protect patients and staff 
(A) Dispensers of alcoholic solutions should be installed in waiting rooms and all patients should be told to use them.

(B) Hemodialysis patients should be told to wash their hands and fistula arm before starting dialysis and should thoroughly disinfect the puncture areas.

(C) Whenever possible, a short evaluation should be made before a patient is allowed to enter the dialysis ward and body temperature should be systematically measured.

(D) Nursing and medical staff working in dialysis rooms should wear surgical masks and protective glasses, wash their hands with soap and water and systematically use alcoholic solutions and disposable gloves. Surgical masks should be changed every 4-6 h, according to type and producer's instructions.

(E) Nursing and medical staff should be tested at least upon the appearance of symptoms, whose definition should be as broad as possible. Testing contacts may be useful, but depends upon availability of the tests.

(F) Patients with a cough, fever (of whatever entity, taking into account the fact that the febrile reaction may be blunted in dialysis patients), upper airway involvement or conjunctivitis should be evaluated before they are allowed to entry into the dialysis ward.

(G) It is advisable that each center organizes an isolated area (unless the hospital has provided an alternative path) for allowing evaluation and testing of these patients.

(H) All patients with suspected signs or symptoms should be tested, keeping in mind the possible subtle manifestation of the disease.

(I) Whenever possible, all patients should wear a surgical mask. In case of shortage, protection should be reserved to

\section{Advice and management of the various possible cases}

Hemodialysis patients from so-called "active" areas, or those who have been in contact with people who tested positive (considering an incubation time of about 15 days):

1. Absence of manifestations of disease:

Besides the rigorous observance of the recommended rules mentioned above, even in settings in which shortage of masks is a problem, these patients should wear a surgical mask from the time they arrive at the center until they leave, therefore for the entire duration of the dialysis session.

In the case of patients who have been in contact with family members or caregivers with suspect or signs of SARS-CoV-2 infection, the minimum requirement is a surgical mask. Testing should be proposed as much as possible, but will be probably decided on a case-by-case basis (availability of the test, risk factors, logistic situation of the dialysis center).

2. Patients with fever and signs of airways infection:

According to the rules defined in each setting, the patient must be sent to the emergency room, or to a dedicated area, isolated from the dialysis ward, for evaluation and work-up before dialysis.

If the infectious disease specialist decides to carry out a nasopharyngeal swab, there are 3 possible situations:

(a) While waiting for the result, the patient should be treated as if affected by SARS-CoV-2;

if dialysis cannot be postponed, whenever possible, the patient must be hospitalized and dialyzed in a room equipped for dialysis treatment. If mobile dialysis water preparation devices are available, they should be previously connected in one or two rooms in the infectious diseases ward or where hospitalization and treatment can be safely carried out. According to the center's experience, a CRRT machine or a portable hemodialysis machine can be considered as alternatives.

For the dialysis care of these cases, health professionals should wear the personal protection advised for infected cases.

(b) If the outcome of the swab is positive, the patient must remain in isolation and the operators in contact with the patient must be equipped as above.

Due to the complexity of the management of dialysis patients, their fragility, their risk of sudden decompensating, we suggest, whenever possible, hospitalizing them until recovery.

Recovery after SARS-CoV-2 infection has to be certified, according to the current indications.

(c) If the outcome of the swab is negative, the patients can return to the dialysis facility.

3. In case of respiratory distress or failure ER staff must be summoned immediately, since the need for mechanical ventilation may be sudden.

4. Patients on peritoneal dialysis should be assisted at home as far as possible, using telereporting assistance or other systems for communication whenever possible (e.g. WhatsApp, video, etc.).

5. It is important to underline that:

In the wait for a curative treatment or an effective vaccine, protection barriers are the best means for minimizing the impact of the epidemic [13].

In case of peripheral centers without the possibility of isolation, and where an infectious disease unit, and an 
intensive care unit are not available, patients with suspect or confirmed disease should be centralized in hospitals that have these requirements.

\section{Conclusions}

Confronting the SARS-CoV-2 outbreak has allowed us to appreciate how efficiently highly-resourced settings can respond to crises. However even such settings are not prepared to deal with the situation, and lessons are only slowly being learnt. There is still an urgent need to accelerate protocols that lead to the implementation of rapid point-of-care diagnostic testing and effective antiviral therapies.

In some high-risk populations, such as dialysis patients, where several individuals are treated at the same time in a limited space and overcrowded areas, our objective must be to ensure protection to patients, the healthcare team and the dialysis ward.

The difficult Italian experience may help other countries to face the challenges. The experience of the Lombardy underlines the need for gathering and sharing our data to increase our knowledge and support common, initially experience-based, and as soon as possible evidence-based position to face this overwhelming crisis.

\section{Compliance with ethical standards}

\section{Conflict of interest None.}

Ethical standard Not required for this paper.

\section{References}

1. https://www.protezionecivile.gov.it/attivita-rischi/rischio-sanit ario/emergenze/coronavirus. Accessed 3 Mar 2020

2. Guan WJ, Ni ZY, Hu Y, China Medical Treatment Expert Group for Covid-19 et al (2020) Clinical characteristics of coronavirus disease 2019 in China. N Engl J Med. https://doi.org/10.1056/ NEJMoa2002032 (epub ahead of print)
3. Zhang W, Du RH, Li B et al (2020) Molecular and serological investigation of 2019-nCoV infected patients: implication of multiple shedding routes. Emerg Microbes Infect 9(1):386-389

4. Bai Y, Yao L, Wei T et al (2020) Presumed asymptomatic carrier transmission of COVID-19. JAMA. https://doi.org/10.1001/ jama.2020.2565

5. Hellewell J, Abbott S, Gimma A et al (2020) Feasibility of controlling COVID-19 outbreaks by isolation of cases and contacts. Lancet Glob Health. https://doi.org/10.1016/S2214-109X(20)30074 -7 (epub ahead of print)

6. Boyce JM, Hymes JL (2018) What we learned from Ebola: preparing dialysis units for the next outbreak. Clin J Am SocNephrol 13(4):669-670

7. Ahmed AE, Alshukairi AN, Al-Jahdali H et al (2018) Development of a risk-prediction model for Middle East respiratory syndrome coronavirus infection in dialysis patients. Hemodial Int 22(4):474-479

8. Peeri NC, Shrestha N, Rahman MS et al (2020) The SARS, MERS and novel coronavirus (COVID-19) epidemics, the newest and biggest global health threats: what lessons have we learned? Int J Epidemiol. https://doi.org/10.1093/ije/dyaa033 (epub ahead of print)

9. Syed-Ahmed M, Narayanan M (2019) Immune dysfunction and risk of infection in chronic kidney disease. Adv Chronic Kidney Dis 26(1):8-15

10. Betjes MG (2013) Immune cell dysfunction and inflammation in end-stage renal disease. Nat Rev Nephrol 9(5):255-265

11. Naicker S, Yang C, Hwang S, Liu C, Jha V (2020) The 2019 Coronavirus epidemics and kidney disease. Kidney Int. https:// doi.org/10.1016/j.kint.2020.03.001

12. Smith N, Fraser M (2020) Straining the system: novel coronavirus (COVID-19) and preparedness for concomitant disasters. Am J Public Health. https://doi.org/10.2105/AJPH.2020.305618 (epub ahead of print)

13. Wilder-Smith A, Freedman DO (2020) Isolation, quarantine, social distancing and community containment: pivotal role for old-style public health measures in the novel coronavirus (2019nCoV) outbreak. J Travel Med. https://doi.org/10.1093/jtm/taaa0 20 (epub ahead of print)

Publisher's Note Springer Nature remains neutral with regard to jurisdictional claims in published maps and institutional affiliations. 